\title{
COMPARING THE EFFECTS OF DIFFERENT TYPES AND LENGTHS OF EXTRACELLULAR DOUBLE-STRANDED RNA MOLECULES IN RAINBOW TROUT CELLS
}

\author{
Sarah Poynter ${ }^{1}$, Brian Dixon ${ }^{1}$, Stephanie DeWitte-Orr ${ }^{2} \S$ \\ ${ }^{1}$ Department of Biology, University of Waterloo, Waterloo, Ontario, Canada, N2L $3 G 1$ \\ 2 Department of Health Sciences and Biology, Wilfrid Laurier University, Waterloo, Ontario, \\ Canada, N2L $3 C 5$
}

\begin{abstract}
Viruses produce double-stranded (ds)RNA during their replicative life cycles; these dsRNA molecules are known as native dsRNA. DsRNA molecules are potent immunomodulators that induce innate immune responses; extracellular dsRNA molecules in mammals are brought into host cells via class A scavenger receptors (SRAs). SRAs have been identified in rainbow trout cells and bind to dsRNA molecules, suggesting these receptors mediate extracellular dsRNA uptake in fish as well. Current research focuses on the synthetic, viral dsRNA mimic, poly I:C, and there are currently no studies using native dsRNA as stimulants for fish cells. Three types of dsRNA were used as stimulants in this study: poly I:C, multiple lengths/sequences of in vitro transcribed dsRNA, and Chum salmon reovirus (CSV) genomic dsRNA. Rainbow trout gonadal cells (RTG-2) were treated with extracellular dsRNA and cellular responses were measured using qRT-PCR for a panel of innate immune genes and an antiviral assay. Rainbow trout gut cells (RTgutGC) and competitive SRA ligands were used to test for the involvement of SRAs in dsRNA binding and uptake. Sequence has little effect on dsRNA-mediated responses in cells, however length appears to be a strong indicator of immunomodulatory potential. Longer molecules induced a stronger response; these length effects were seen with all three dsRNA molecules. As is seen in mammals, extracellular dsRNA entry in fish cells is mediated by SRAs and involves clathrin-mediated endocytosis. A more thorough understanding of how host cells recognize and respond to biologically relevant dsRNA has applications in adjuvant and antiviral therapy design.
\end{abstract}

\section{KEYWORDS}

Rainbow trout, innate immunity, dsRNA, viruses, scavenger receptors

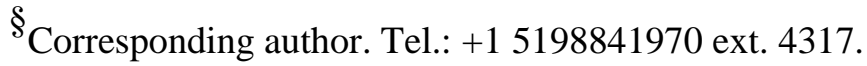

E-mail address: sdewitteorr@wlu.ca 